\title{
An inexpensive contact-sensing device
}

\author{
PHILLIP S. LASITER \\ Arizona State University, Tempe, Arizona 85281
}

\begin{abstract}
A detector is described that utilizes phase-shift techniques for the measurement of contact responses.
\end{abstract}

The present article describes a contact detector that may be utilized for recording applications that cannot be accommodated by simple mechanical switching devices. The low unit cost and universal aspects of the device permit the experimenter to measure various behaviors more readily in experimental situations.

The simple contact-sensing circuit is shown in Figure 1. The circuit is designed around a LM-567 tone decoder. The decoder's free-running frequency at pin 6 is coupled to the input terminal (pin 3) of the device. This configuration allows the output of the VCO at pin 5 to be in phase with the input at pin 3, which prevents the circuit from locking. Whenever a sufficient mass is in contact with the sensing plate, $\mathrm{P1}$, the center frequency of the input terminal is shifted out of phase, allowing the circuit to lock. The output is then driven high through the pull-up resistor $(1 \mathrm{~K})$, providing a signal source through the opto coupling device. The sensitivity of the unit is varied by the $500 \mathrm{~K}$ potentiometer that adjusts the input signal level at pin 3 .

The center frequency of the tone decoder is equal to the free-running frequency of the VCO. This may be determined by the relationship:

$$
\mathrm{f}_{\mathrm{o}} \cong 1 / \mathrm{R}_{\mathrm{t}} \mathrm{C}_{\mathrm{t}},
$$

where $R_{t}$ and $C_{t}$ are the component values at pins 5 and 7. The bandwidth of the decoder may also be found by the approximation:

$$
B W=1070 \sqrt{V_{\text {in }} / f_{o} C_{3}} \text { in } \% \text { of } f_{o}
$$

where $V_{\text {in }}$ is equal to the input voltage $\left(V_{\text {in }}<200 \mathrm{mV}\right)$ and $C_{3}$ is equivalent to the component value at pin 2 . These parameters define the time required for the tone decoder to lock, given contact. The number of cycles $\left(f_{o}\right)$ necessary to lock may be approximated by:

$$
\mathrm{C}_{\mathrm{L}}=350(\mathrm{BW})^{-1.15}
$$

where $C_{L}$ is the number of cycles and $B W$ is in terms of percent of $f_{0}$. The minimum on-off cycling rate is given as:

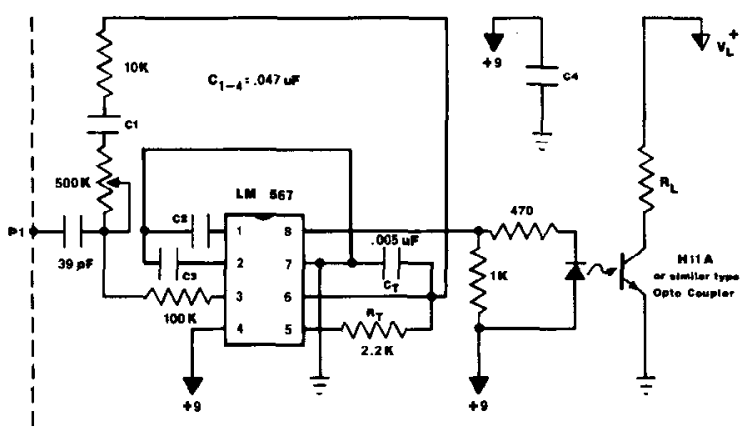

Figure 1. Contact-detector circuit.

$$
f_{0} / 20
$$

For the component values described in Figure $1, f_{o}$ is approximately $90 \mathrm{kHz}$ and the $\mathrm{BW}$ ranges between $2 \%$ and $8 \%$. These values provide lock times between $2 \mathrm{msec}$ and 500 microsec, respectively, and approximately 5 -msec on-off cycling time. Alternate component values for other applications may be selected by utilizing the above equations.

The contact plate P1 must be constructed of materials that will not deteriorate under adverse conditions, and they should be located as close as possible to the detector for ideal operation. Minimizing the plate area also reduces spurious noise problems. Stainless steel provides excellent contact plates, although oily deposits left by rodent subjects must be cleaned occasionally for proper operation. The circuit can be constructed on a $7.5 \times 7.5 \mathrm{~cm}$ circuit board; wiring loops should be avoided in the area near the input terminals and coupling capacitor ( 39 picoF). Portions of chamber walls, drinking tubes, floor grids, or other areas of interest may be coupled to the contact device. Because the sensor does not rely on current passed through the subject, the contact detector may be a viable alternative to standard lickometers. The components cost approximately $\$ 10$.

(Received for publication October 17, 1978; revision accepted December 14,1978 .) 